\title{
DRG: ne pas leur faire faire ce qu'ils ne savent pas faire
}

Le $1^{\text {er }}$ juin dernier, le peuple suisse a rejeté un article constitutionnel 117a qui augurait de mutations dans la manière de pratiquer la médecine. Pour le financement des soins hospitaliers, une modification majeure est en route, avec notamment la création en janvier 2008 de la société d'utilité publique SwissDRG, portée sur les fonts baptismaux par plusieurs partenaires dont la FMH. Il s'agit (de manière généralisée dès 2012) de payer les soins hospitaliers en fonction du «portrait thérapeutique» des soins requis par l'affection traitée. L'idée étant que les prestations que nécessitent les atteintes aiguës sont suffisamment semblables et codifiées pour que la caractérisation par DRG (Diagnosis Related Groups) soit une base adéquate pour le remboursement des frais. Une telle rétribution doit avoir un effet motivant, dans le sens de l'efficience, sur les prestataires.

A ce stade, le système est prévu pour les soins somatiques aigus et il importe de voir qu'il n'apparaît pas approprié dans d'autres domaines. Ainsi par exemple pour les soins palliatifs, qui représentent un changement de paradigme: ce n'est plus la guérison, ou une vie dans de bonnes conditions à long terme, qui est l'objectif, mais le meilleur accompagnement de ceux dont la mort est proche. Ce n'est plus la nature de la maladie qui est déterminante mais soulager et être aux côtés du malade, pour une durée difficile à fixer a priori. L'usage d'un DRG standard pousserait à des soins palliatifs expéditifs et mettant l'accent sur la technique, ce qui n'est pas leur vocation. Des considérations similaires valent pour la psychiatrie.

Un souci des médecins et soignants est que les DRG donnent lieu à une alliance objective entre assureurs et directions hospitalières, directions qui auront des résultats financiers d'autant meilleurs que les séjours seront plus courts. D'où le risque de ce qu'on dénomme en allemand «blutige Entlassungen» («sorties saignantes»). Des changements dans les loyautés et collaborations qui valaient jusqu'ici sont ainsi vraisemblables. C'est une fatalité que tout système induit des modifications de comportement des «acteurs rationnels» que sont les parties concernées: chacun tend à utiliser les caractéristiques du système en maximisant son intérêt. Il n'y a pas lieu ici de condamner mais il faut en être conscient et mettre en place des checks and balances contrant les effets indésirables. Un certain nombre de cantons déjà, romands et alémaniques, utilisent complètement ou partiellement des systèmes DRG dans leurs hôpitaux (ainsi, l'Ente ospedaliero cantonale tessinois depuis sept ans) et ont introduit des contrôles de qualité (qualité des soins, de l'enregistrement et du codage - confiant le codage à des spécialistes indépendants des équipes soignantes). Un projet de l'Institut d'éthique biomédicale de Zurich entend investiguer les dimensions éthiques de la problématique [1].

Malgré ces craintes et un certain scepticisme, on ne saurait s'opposer à de nouvelles méthodes de gestion, compte tenu d'évolutions comme le caractère de plus en plus technique et le raccourcissement des séjours - et, dans le cas particulier, d'une décision parlementaire. Mais il importe de maintenir dans ce pays une «culture des soins» conforme à ce que la population attend, et d'éviter que les DRG deviennent le lit de Procuste sur lequel tous les séjours en établissement sanitaire seraient mesurés et remboursés. D'autres modèles restent indispensables: quand les soins infirmiers ou de base et des soutiens non médicaux sont au premier plan; quand la prise en charge ne dépend pas au premier chef du diagnostic mais de l'état général du malade (marqué par des polypathologies très diverses, qui ne sont pas aisément définies) et de ses besoins dans la vie quotidienne. Ainsi - sous réserve d'élaboration de modèles très adaptés - il faudra résister à l'introduction des DRG dans les prises en charge chroniques, dans les institutions pour personnes dépendantes, âgées ou handicapées, dans les soins à domicile.

$\mathrm{Au}$ soir du $1^{\mathrm{er}}$ juin, la Société vaudoise de médecine saluait le «retour des médecins en politique». Dans la mise en œuvre des DRG, les praticiens ont le devoir de mettre le doigt sur de possibles conséquences préoccupantes voire perverses. Tout en tirant avantage du modèle là où il est pertinent, son application ne doit pas, ailleurs, pousser à des prises en charge technicisantes, insuffisamment humanisées et de trop
Jean Martin*

\section{courte durée.}

Jean Martin \footnotetext{
de la Commission nationale de la Commis
d'éthique.

* Le Dr Jean Martin, membre de la rédaction, est ancien médecin
} 